\title{
Peripheral Nerve Imaging
}

\author{
James F. Griffith and Roman Guggenberger
}

\section{Learning Objectives}

- To understand the ultrasound and MRI appearances of different nerve pathologies.

- To understand the complementary roles of US and MRI in evaluating different nerve pathologies.

\subsection{Introduction}

The main peripheral nerve abnormalities that lead to referral for imaging are, in order of frequency: entrapment, tumours, trauma, perineural fibrosis, neuralgic amyotrophy, and inflammatory neuropathy. These conditions are best imaged with either ultrasound or magnetic resonance imaging (MRI). Both modalities allow nerves to be seen in high resolution and have their own advantages that make them complimentary in peripheral nerve imaging. Ultrasound is more readily available, allows large segments of nerves to be imaged relatively quickly, and has a dynamic component. MR neurography (MRN) provides an excellent overview of the neural anatomic environment; enables more consistent visualisation of the smaller peripheral nerves, such as those in the foot; can visualise nerves in areas where transducer access is limited, such as the intraforaminal part of the brachial plexus; is less operator dependent; enables the functional capacity of nerves to be explored in greater depth through, for example, diffusion tensor imaging; and allows the effect of motor denervation, i.e. muscle atrophy, to be seen and quantified more accurately.

J. F. Griffith $(\triangle)$

Department of Imaging and Interventional Radiology, The Chinese University of Hong Kong, Shatin, Hong Kong

e-mail: griffith@cuhk.edu.hk

R. Guggenberger

Institut für Diagnostische und Interventionelle Radiologie,

Universitätsspital Zurich, Zurich, Switzerland
This chapter discusses imaging of the peripheral nerves by ultrasound and MRI.

\subsection{Peripheral Nerve Basics}

Peripheral nerves range in diameter from 1 to $20 \mathrm{~mm}$ and are formed by multiple axons, grouped into fascicles which, in turn, are grouped into nerves. The endoneurium is the connective tissue surrounding the axon-Schwann cell unit and contains collagen fibres, fibroblasts, capillaries, and a few mast cells and macrophages. The perineurium binds groups of axon-Schwann cell units into nerve fascicles. The number and size of the nerve fascicles generally diminish as the nerve gets smaller more peripherally. The epineurium is the outermost connective tissue sheath that encloses all the nerve fascicles, providing mechanical support to the nerve and reducing friction with adjacent tissues [1]. The endoneurium and perineurium form a blood-nerve barrier akin to the blood-brain barrier. As such, peripheral nerves, except for the dorsal nerve root ganglia, do not enhance with intravenous contrast unless the barrier is compromised by inflammation or malignancy [2].

\subsection{Pathophysiology Basics}

Pathological nerves may show neural \pm fascicular oedema, altered calibre, irregular contour, discontinuity, abnormal enhancement, perineural fibrosis, or rarely intraneural fatty replacement $[3,4]$. In acute nerve pathologies, fascicular pattern is usually accentuated due to intra- and perineural oedema, leading to increased nerve calibre and signal intensity. With increasing chronicity, oedema subsides and fibrotic nerve changes lead to reorganisation of nerve tissue with decreased nerve diameter and loss of T2-hyperintensity on MRI. MR imaging is more sensitive at detecting target muscle denervation oedema or atrophy than ultrasound. Diffuse 
muscle oedema and contrast enhancement can be seen within 1 day of denervation. Both probably reflect increased muscle engorgement and increased muscle blood volume due to denervation. Muscle atrophy and fatty replacement are features of chronic denervation.

\subsection{MR Neurography: Technical Considerations}

MR neurography (MRN) aims to provide high-resolution high-contrast images of nerves. This is aided by suppression of the background fat as well as pulsation and breathing artefact suppression. While homogeneous fat suppression is challenging on $3 \mathrm{~T}$ imaging, the increase in contrast-to-noise ratio and spatial resolution achieved by the higher field strength outweigh the issues with inadequate fat suppression. Dedicated peripheral coils ensure high-quality nerve detail and can, if necessary, be combined with surface coils when larger sections of the nerve need to be examined. For example, the brachial plexus is usually imaged with a combined head-neck coil to cover the cervical spine, foramina, and neck regions along with a torso coil to cover the parascapular and axillary regions.

Since nerves have a long (e.g. $80-100 \mathrm{~ms}$ ) T2 signal and are usually surrounded by fat, fast or turbo spin-echo sequences with spectral saturation using the adiabatic inversion pulse and Dixon technique are preferred [5]. The Dixon technique allows larger areas to be imaged with homogenous fat suppression when, for example, imaging the brachial or lumbosacral plexuses.

Focal T2-weighted neural hyperintensity may occur if the course of the nerve changes relative to the B0-field into a plane higher than $55^{\circ}$. This is known as magic angle artefact which may also persist at TEs beyond $66 \mathrm{~ms}$. Mild T2-hyperintensity of nerves can be seen in asymptomatic subjects [6]. If in doubt regarding the significance of any neural hyperintensity, comparison with the contralateral healthy side is helpful, as is assessing the target muscles for signs of denervation. Also, true pathologic intraneural T2-hyperintensity is usually higher than any maximal magic angle effect [7].

Recent advancements with extended echo train lengths and parallel imaging have enabled 3D T2-weighted imaging volumetric visualisation of nerves using fast or turbo spinecho sequences in acceptable scan times [8, 9]. Suppression of adjacent vasculature can be achieved by motion-sensitised driven equilibrium or diffusion moment, improving the discrimination of small nerves in the extremities and skull base [10-12]. Contrast administration is not routinely used when imaging peripheral nerves though it can help to enhance vessel conspicuity and demonstrate inflammatory or neoplastic uptake on T1-weighted fat-suppressed images as well as simultaneously suppressing vessel signal on T2-weighted spin-echo and STIR images to increase neural delineation.

Diffusion tensor imaging (DTI) provides additional information on nerve integrity and can be a useful adjunct to standard MRN protocols. Random multidirectional motion of water molecules is restricted when the highly organised neural membranes are intact. The amount of restriction can be quantified by apparent diffusion coefficient (ADC) which is an estimate of mean water diffusivity in all directions. Diffusion can be further directionally quantified by fractional anisotropy with 0 being full isotropy and 1 being full anisotropy. Diffusion tractography, by connecting voxels of similar diffusivity, helps visually confirm neural integrity [13].

\section{Key Point}

- The highly organised structure of peripheral nerves is similar throughout the peripheral nervous system. Nearly all nerves look the same. Similarly, the ultrasound and MRI appearances of entrapment, tumour, injury, or inflammation tend to be similar irrespective of the nerve(s) affected.

\subsection{Neural Entrapment}

Carpal tunnel syndrome (CTS) is the commonest nerve entrapment. The main ultrasound and MR criterion of CTS is undue swelling of the median nerve. The carpal tunnel inlet and outlet are determined on ultrasound and MRI by direct visualisation of the transverse retinaculum. On ultrasound and MRI, one should measure the cross-sectional area (CSA) of the median nerve at the distal forearm, just proximal to the tunnel, at the tunnel inlet, at the tunnel outlet, and just distal to the tunnel.

The more swollen the median nerve, the more likely the possibility of CTS. The normal median nerve CSA at any of these locations on ultrasound is $<10 \mathrm{~mm}^{2}$. A median nerve CSA of $>14 \mathrm{~mm}^{2}$ at any of these locations is diagnostic of CTS [14]. Measurements between 10 and $14 \mathrm{~mm}^{2}$ should be taken as suggestive of, but not diagnostic of, CTS. Ultrasound CSA measurements do not include the epineurium, and, hence, MRI measurements are about $20 \%$ greater than ultrasound measurements [15]. On MRI, a median nerve CSA $>15 \mathrm{~mm}^{2}$ either proximal to or distal to the tunnel is a useful diagnostic criterion for CTS while a CSA > $19 \mathrm{~mm}^{2}$ proximal to the tunnel is indicative of severe CTS [16]. Intraneural hyperaemia on ultrasound is a specific but insensitive ultrasound sign of neural compression. Other ultrasound and MR signs of CTS such as increased palmar retinacular bowing or retinacular thickness, loss of neural 
fasciculation, flattening ratio, and reduction in carpal tunnel CSA are not specific enough to be very discriminatory on a case-by-case basis in everyday clinical practice.

Ultrasound is as sensitive as nerve conduction studies in confirming the presence of CTS. Patients with equivocal nerve conduction study findings for CTS will also usually have equivocal findings on ultrasound so the two examinations are not necessarily complimentary. Elastography and DTI studies have shown that CTS patients slower elasticity (i.e. stiffer) and median nerves with lower fractional anisotropy values (i.e. less unidirectional diffusion) though currently elastography and DTI do not provide additional information over that provided by CSA measurements alone.

Primary CTS is more common than secondary CTS due to, for example, tenosynovitis, ganglion cysts or other carpal tunnel masses, or crystal deposition within the carpal tunnel. All of these secondary causes can be screened with ultrasound or MRI, with MRI being particularly helpful in this regard.

Following carpal tunnel release, the median nerve will usually remain unduly swollen for at least 1 year following successful surgery and may never return to normal size despite good amelioration of symptoms [17]. This persistent median nerve swelling is due to neural fibrosis and congestion secondary to chronic compressive neuropathy. Therefore, a swollen median nerve following surgery on ultrasound or MRI should not be taken as a sign of incomplete decompression. Similarly, the retinaculum will reform in most patients at 3-12 months following surgery, though in a more elongated fashion such that the overall volume of the carpal tunnel is increased [17].

The next most common entrapment is cubital tunnel syndrome which occurs due to compression of the ulnar nerve either within the cubital tunnel deep to Osborne's ligament or within the cubital tunnel proper deep to the arcuate ligament. Any imaging of the cubital tunnel should extend from $8 \mathrm{~cm}$ proximal to the elbow joint, to include the arcade of Struthers, to $5 \mathrm{~cm}$ distal to the joint to include the ulnar nerves between the two heads of flexor carpi ulnaris [18].

Primary cubital tunnel syndrome is less common than secondary cubital tunnel syndrome due to, for example, a prominent anconeus epitrochlearis muscle osteophytosis, synovitis, or ganglion cysts encroaching from the ulnohumeral joint into the cubital tunnel. Secondary cubital tunnel syndrome tends to be mainly seen in older patients.

The greater the ulnar nerve CSA in the cubital tunnel, the more likely the diagnosis of cubital tunnel syndrome. The normal ulnar nerve CSA is $<8 \mathrm{~mm}^{2}$. An ulnar nerve CSA $>14 \mathrm{~mm}^{2}$ at the cubital tunnel is considered diagnostic of cubital tunnel syndrome. CSA values between 8 and $12 \mathrm{~mm}^{2}$ are suggestive of, but not diagnostic of, cubital tunnel syndrome.
Ultrasound also allows one to examine for ulnar nerve subluxation during elbow flexion more easily than MRI. Subluxation occurs due to a redundancy of Osborne's ligament. Although ulnar nerve subluxation is usually asymptomatic and can be seen in about one-fifth of healthy subjects, one can still appreciate how repeated subluxation of the ulnar nerve over the medial humeral epicondyle could lead to friction neuritis ('ulnar neuritis') in patients who frequently flex their elbows during daily, occupational, or sporting activities.

Although not discussed much in the literature, it is likely that two pathologies, namely, neural compression and inflammation, may act in isolation or in unison to produce symptoms of cubital tunnel syndrome. Some patients may have an inflamed ulnar nerve which is not compressed. This is different to other entrapment syndromes such as carpal tunnel syndrome, where the primary aetiology is solely that of neural compression. In this respect, MR has an advantage over ultrasound in allowing one to determine neural oedema by T2-hyperintensity. Adjacent muscle acts as an internal reference to standardise ulnar nerve signal intensity [19]. Then measure the contrast-to-noise ratio (CNR) as follows:

$\mathrm{CNR}=\frac{\text { Ulnar nerve signal intensity }- \text { muscle signal intensity }}{\text { Standard deviation of air }}$

A CNR of $>50$ has high accuracy for has high accuracy for cubital tunnel tunnel syndrome.

The third most common entrapment syndrome is supinator syndrome where the motor branch of the radial nerve (the posterior interosseous nerve) is entrapped at the inlet or just beyond the inlet of the supinator tunnel, known as the arcade of Frohse (Fig. 18.1). One will appreciate undue swelling of the deep branch of the radial nerve just proximal to or within the supinator tunnel. There is often also localised deep tenderness at this point.

Less frequent sites of neural entrapment include the suprascapular groove, the spinoglenoid notch (Fig. 18.2), the radial groove, the radial tunnel, Guyon's canal, the peroneal canal, and the tarsal tunnel.

\section{Key Points}

- Entrapment syndromes are common in the upper limb, uncommon in the lower limbs.

- At the relevant sites, the greater the degree of nerve swelling, the more likely the possibility of entrapment.

- The CSA of peripheral nerves on MRI is $20 \%$ larger than on ultrasound.

- Both ulnar nerve entrapment and friction neuritis can produce cubital tunnel syndrome symptoms. 

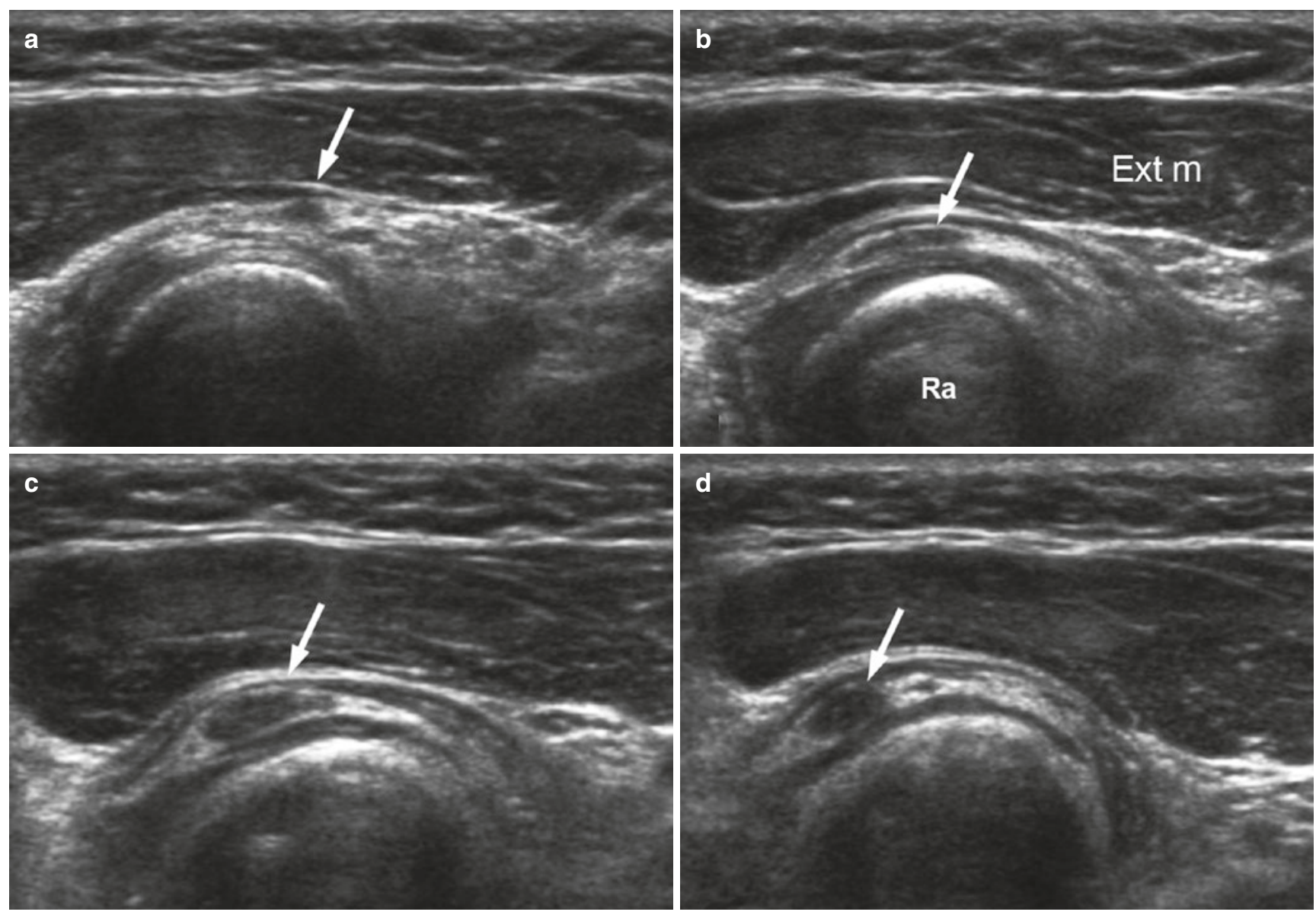

Fig. 18.1 Series of transverse ultrasound images showing posterior interosseous nerve (arrows) (a) proximal to and (b-d) within the supinator tunnel of a 9-year-old boy with quite abrupt onset of weakness of finger extension. The PIN is swollen within the supinator muscle ('supinator tunnel') located between the radial neck and the extensor forearm

\subsubsection{Tumours}

More than $90 \%$ of peripheral nerve tumours are benign and more than $60 \%$ of these benign nerve sheath tumours are schwannomas. The next most common benign nerve sheath tumour is neurofibroma. Other benign nerve sheath tumours include intraneural lipoma and intraneural ganglia.

Schwannomas arise from Schwann cells, while neurofibroma comprises a mixture of neural cells. There are no known imaging features that allow one to accurately distinguish between a neurofibroma and schwannoma. As such, one should refer to the presence of a 'nerve sheath tumour' rather than a particular tumour type. Neural continuity is the most specific ultrasound or MR sign of a nerve sheath tumour (Fig. 18.3). Other less specific ultrasound or appearances are a fusiform or oblong-shaped mass, internal cystic, myxoid or haemorrhagic areas, posterior acoustic enhancement (on ultrasound), and moderate tumoural hyperaemia or enhancement. On MRI, the so-called target sign may be more sug- muscles. This swelling was due to an hourglass constriction at the arcade of Frohse confirmed surgically. PIN swelling is more commonly seen immediately proximal to the supinator tunnel inlet. Ext $\mathrm{m}$ Extensor carpi radialis brevis muscle

gestive of a schwannoma, while more fascicular patterns are seen with neurofibromas. Only about $70 \%$ of nerve sheath tumours have visible neural continuity on ultrasound examination, and it is only in this setting that one can make a definitive diagnosis of a nerve sheath tumour based on the ultrasound appearances alone. When neural continuity is present, one should ascertain whether the tumour is located eccentric or concentric to the parent nerve as this has a bearing on the success of surgical excision. Percutaneous biopsy of nerve sheath tumour is occasionally very painful and should not be undertaken when a confident diagnosis can be made based on ultrasound appearances alone provided malignancy is not suspected.

The remaining 30\% of nerve sheath tumours do not have imaging appearances specific enough to make a definitive diagnosis based on ultrasound appearances alone. In this situation one can offer a probable or possible diagnosis of a nerve sheath tumour. Potential differential diagnoses are vascular leiomyomas, vascular malformation, glomus, fibroma, 

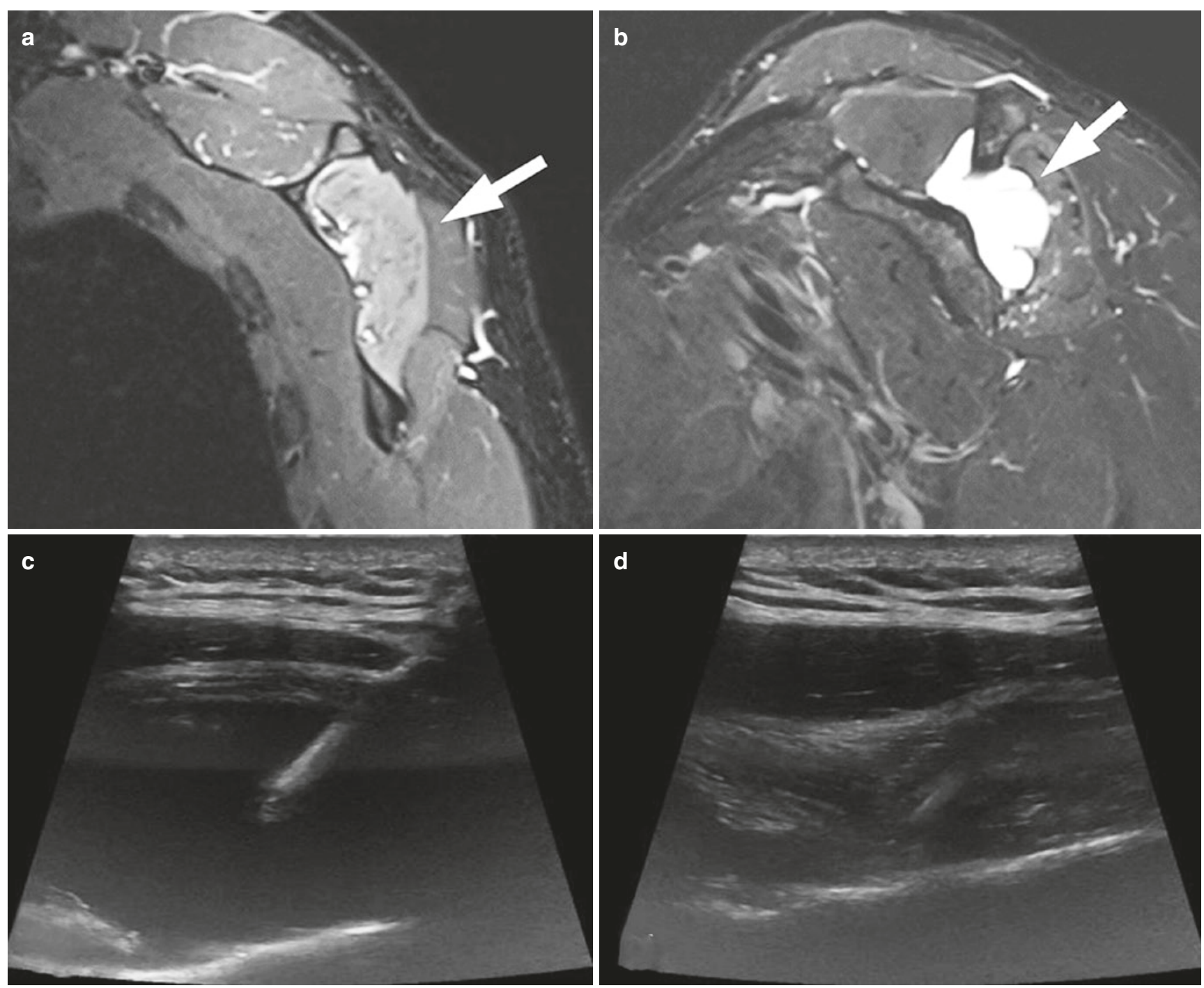

Fig. 18.2 Adult patient with increasing shoulder weakness while working overhead in vineyard. (a) MRN shows moderate infraspinatus denervation oedema (arrow) with (b) a large paralabral ganglion

and ganglia. Appearances that would favour a nerve sheath tumour over these tumours are an oblong configuration to the mass, a regular margin, more pronounced internal speckling, and lack of vascular convergence.

Less common types of neurofibroma are diffuse and plexiform neurofibromas. Diffuse neurofibroma may be sporadic or associated with neurofibromatosis type I (NF1). They originate from the subcutaneous nerves and spread extensively along the subcutaneous connective tissue septae. One will see localised dermal and subcutaneous thickening, subcutaneous reticulation, and hyperaemia which can mimic localised cellulitis or panniculitis. Plexiform neurofibroma is only seen in NF1 and is associated with a moderate risk of malignant transformation. It

(arrow) causing suprascapular nerve compression in the spinoglenoid notch. This compression was relieved by US-guided cyst aspiration as seen on (c) pre- and (d) post-aspiration images

has a more distinctive 'bags of worms' appearance with diffuse enlargement of nerves in a discrete lobulated mass and may occur in the subcutaneous as well as the deep tissues.

One in two malignant peripheral nerve sheath tumours (MPNST) occur in patients with NF1, while 1 in 20 patients with NFI will develop a MPNST. There are currently no imaging features that allow one to distinguish atypical nerve sheath tumours from MPNSTs [20] (Fig. 18.4). Both wholebody MRI with diffusion-weighted imaging and PET/CT imaging are currently being explored in this regard [20]. If there is clinical or imaging suspicion of MPNST, then a percutaneous biopsy should be undertaken to confirm or refute this possibility. 


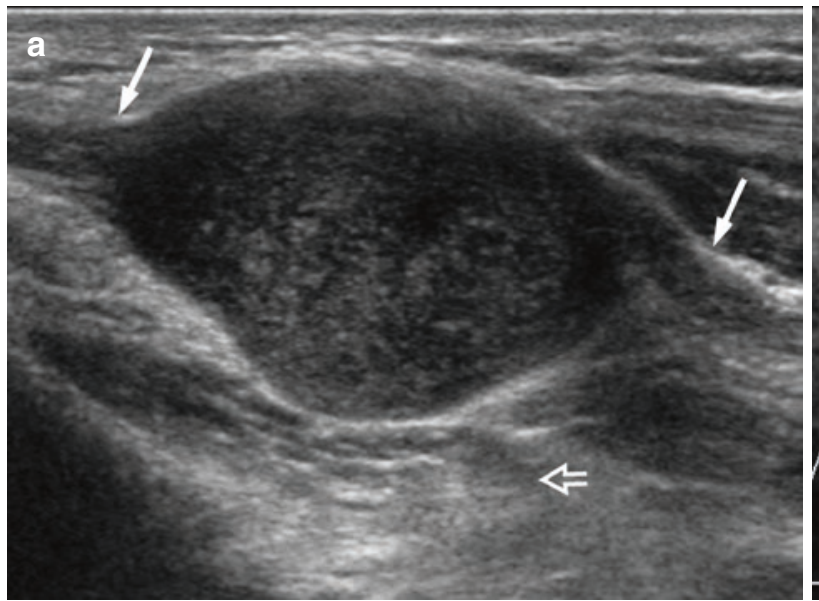

Fig. 18.3 Longitudinal ultrasound of distal forearm showing (a) medium-sized nerve sheath tumour arising eccentrically from the median nerve (arrows) with neural continuity and acoustic enhance-

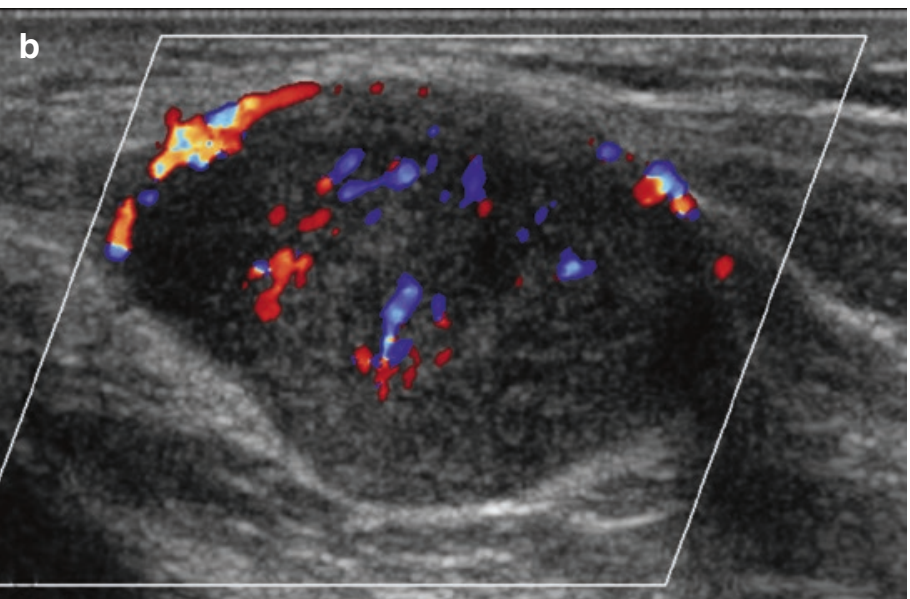

ment (open arrow). (b) The tumour shows moderate tumoural hyperaemia. These are diagnostic ultrasound appearances of a nerve sheath tumour
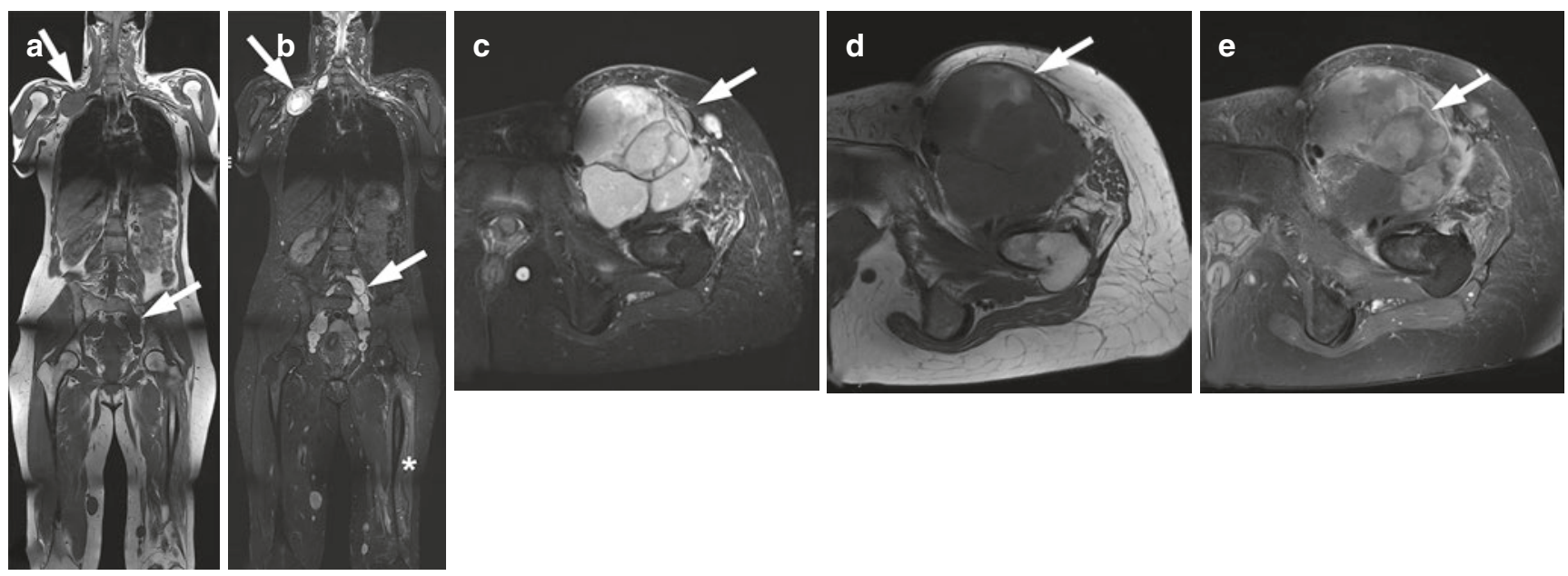

Fig. 18.4 Middle-aged women with hereditary schwannomatosis. (a) Multiple tumours are seen on T1-weighted and (b) T2-weighted STIR images (arrows). Severe denervation oedema and atrophy of the left quadriceps muscle is present (asterisk in b) as a consequence of a large

\section{Key Points}

- About $70 \%$ of nerve sheath tumours have neural continuity evident on imaging. Only in this setting, can one make a definite diagnosis based on imaging signs alone.

- Diagnostic accuracy of imaging for identifying nerve sheath tumours is much less when no neural continuity exists.

- Increase in size over time is the most reliable sign of the MPNST. If doubt exists, percutaneous biopsy is warranted. dedifferentiated malignant schwannoma (confirmed histologically) of the femoral nerve with inhomogeneous signal on (c) T2-weighted STIR, (d) T1-weighted, and (e) T1-weighted fat saturated post-gad images

\subsubsection{Nerve Injury}

Blunt injury to nerves usually recovers spontaneously and is not imaged. Conversely, sensory or motor denervation following a penetrating injury usually results in early surgical exploration without the need for imaging.

Imaging is, therefore, usually undertaken to investigate either traction injury or the delayed recognition of nerve injury following penetrating trauma. In traction injury, imaging should focus on those nerves which have been clinically compromised, while in penetrating trauma, imaging should focus on examination of those nerves close to the path of injury. A neuroma-in-continuity (NIC) or stump neuroma 
can be seen some months following partial or complete nerve transection, respectively, and should not be mistaken for a nerve sheath tumour [21].

Features of nerve injury to ascertain on imaging are:

(a) Is the nerve visibly injured, i.e. is it not swollen or mild/ moderately/severely swollen? What is the length of the affected segment? Is there perineural fibrosis, haematoma, or inflammation present? Is there neural adhesion? If perineural fibrosis is present, what percentage of the neural circumference is affected?

(b) Is there neural transection present and, if so, is this complete or incomplete?

(c) If neural transection is incomplete: what is the approximate percentage of neural transection. What is the length (in $\mathrm{mm}$ ) of the gap between the retracted fibres? Is there any fibrosis or neuroma-in-continuity (NIC) present? What is the size of this NIC?

(d) If neural transection is complete: What is the length (in $\mathrm{mm}$ ) of the gap between the retraced nerve endings? Is there any end fibrosis or end neuroma present at the cut ends of the nerve? If present, what is the size of the neuroma? What is the distance between the visibly normal sections of nerve at either end of the transection? (Fig. 18.5)

Peripheral nerve damage is usually graded according to Seddon and Sunderland, where corresponding signal changes can be seen on MRN (Table 18.1).
However, the Sunderland classification was devised on the basis of neural crush injury. Hence it does not apply to traction injury or penetrating injury which is the most common in practice. It does not, for example, include incomplete neural transection which is a common traumatic neural injury.

\section{Key Points}

- Most neural injury does not require imaging.

- The main points to consider are as follows: (1) is the nerve injured, i.e. swollen or with altered echotexture or signal intensity; (2) is there neural transection present and, if so, is it partial or complete; and (3) is there a neuroma present.

- A normal looking nerve or a mildly abnormal nerve on imaging has a good clinical outcome.

Table 18.1 Sunderland grade with commonly used terminology, neural integrity, and MR appearances

\begin{tabular}{|c|c|c|c|}
\hline Grade & Terminology & Integrity & MRN \\
\hline I & Neuropraxia & Intact & Hyperintense nerve \\
\hline II & $\begin{array}{l}\text { Axonotmesis } \\
\text { (mild) }\end{array}$ & Axons torn & $\begin{array}{l}\text { Hyperintense, thickened } \\
\text { with prominent fascicles }\end{array}$ \\
\hline III & $\begin{array}{l}\text { Axonotmesis } \\
\text { (moderate) }\end{array}$ & $\begin{array}{l}+ \text { Endoneurium } \\
\text { torn }\end{array}$ & \\
\hline IV & $\begin{array}{l}\text { Axonotmesis } \\
\text { (severe) }\end{array}$ & $\begin{array}{l}+ \text { Perineurium } \\
\text { torn }\end{array}$ & Heterogeneous nerve \\
\hline V & Neurotmesis & $\begin{array}{l}+ \text { Epineurium } \\
\text { torn }\end{array}$ & $\begin{array}{l}\text { Complete nerve } \\
\text { transection }\end{array}$ \\
\hline
\end{tabular}
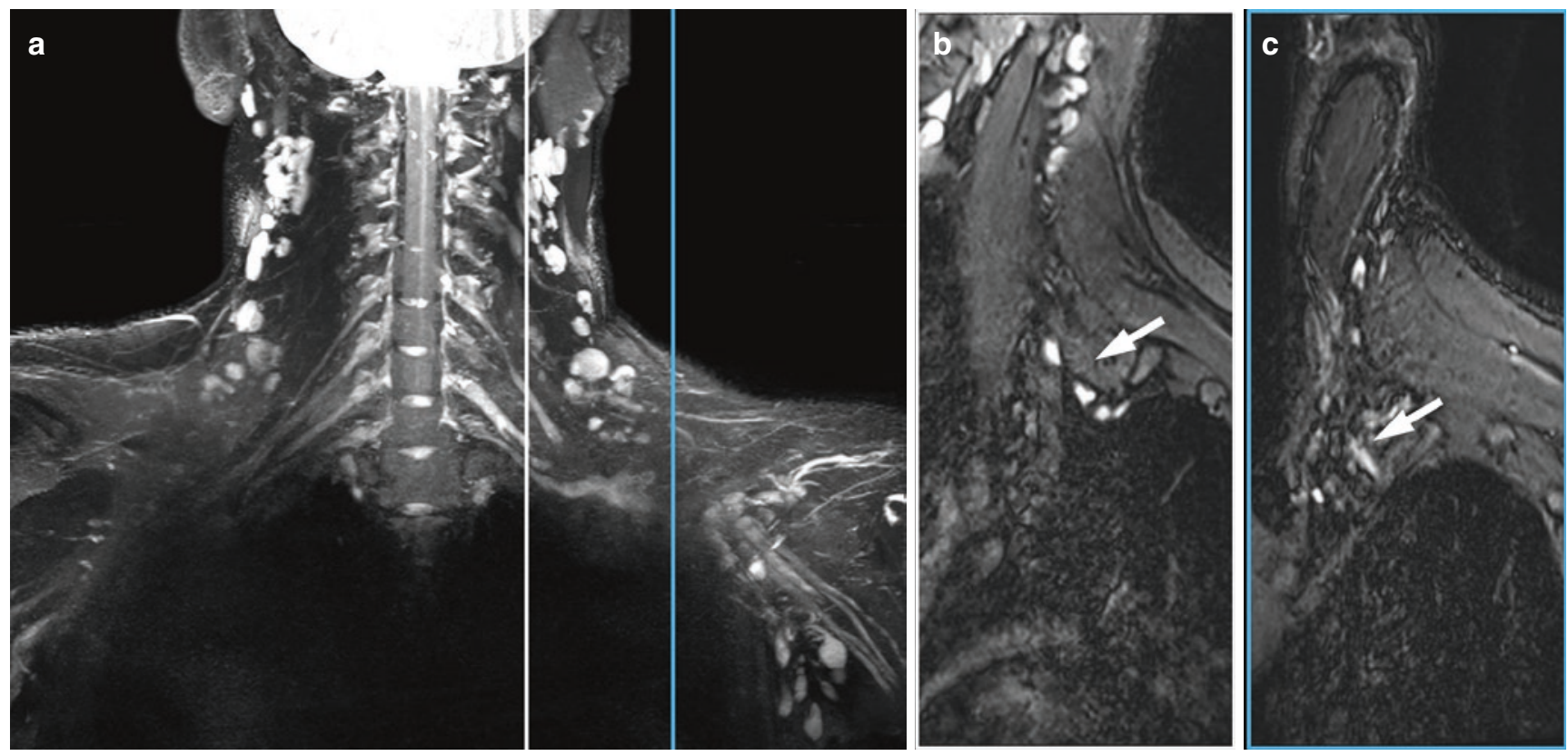

Fig. 18.5 Young man with transection of the entire left brachial plexus following an avalanche injury. (a) Coronal MIP of 3D SPACE STIR shows enlarged, retracted nerve stumps with (b) small stump neuroma formation (arrow) and (c) missing plexal structures more laterally (arrow) 


\subsubsection{Perineural Fibrosis}

Perineural fibrosis is nearly always related to either prior radiation, surgery, or trauma. The most common site of postirradiation perineural fibrosis is the brachial plexus, following irradiation for breast or nasopharyngeal carcinoma, usually with a 1-2-year latent period before symptoms emerge. The ultrasound and MR appearances of brachial plexopathy are as follows: increased size of the neural elements, poor delineation, obliterated or 'dirty' perineural fat, clumping or adhesions of the neural elements, intraneural or perineural hyperaemia/enhancement, and distortion of the neural and perineural tissues.

The main differential diagnosis, both clinically and on imaging, is metastatic infiltration of the brachial plexus. Metastatic infiltration of the brachial plexus is often, though not always, a feature of end-stage metastatic breast, head and neck, or lung carcinoma and appears, on imaging, as a discrete, often hyperaemic, mass infiltrating or adjacent to the brachial plexus. One may sometimes see metastatic infiltration of adjacent structures, such as the vertebrae or lung apices, as well as accompanying metastatic-type adenopathy. Occasionally, percutaneous biopsy may be necessary to distinguish between nodular post-irradiation fibrosis and metastatic disease. In such cases, this biopsy can be performed safely under ultrasound guidance.

\section{Key Points}

- Radiation is the most common cause of peripheral perineural fibrosis with the brachial plexus being most affected.

- Imaging appearances of perineural fibrosis are increased size, poor delineation, 'dirty' perineural fat, adhesion, hyperaemia/enhancement, and perineural distortion.

\subsubsection{Neuralgic Amyotrophy}

Neuralgic amyotrophy (or Parsonage-Turner syndrome) is preferable a term to 'brachial neuritis' as the nerves beyond the brachial plexus are not infrequently affected. Neuralgic amyotrophy is an inflammatory condition of unknown aetiology which, as the name implies, leads to both severe pain and muscle weakness, particularly in the periscapular region though also more peripherally in the upper limb. There is often a preceding trigger such as infection or vaccination. Neuralgic amyotrophy may wax and wane over several months with different nerves being affected. Usually only one side is affected, and even when both upper limbs are affected, it is typically sequential rather than simultaneous. The mixed suprascapular nerve is the most frequently affected nerve. A suprascapular nerve CSA of $>4.2 \mathrm{~mm}^{2}$ on ultrasound has a sensitivity of $86 \%$ and a specificity of $92 \%$ for neuralgic amyotrophy.

MRI is excellent at demonstrating the atrophic and denervation changes in the parascapular and other muscles (Fig. 18.6). Ultrasound, on the other hand, is particularly advantageous in allowing ready examination of potentially affected nerves beyond the brachial plexus. Extraplexal nerves that tend to be most affected are the long thoracic, axillary, median, and radial nerves as well as the anterior and posterior interosseous nerves. Affected nerves may show localised neural swelling \pm neural fascicular entwinement \pm hourglass constriction. These features can be appreciated on US and MR. The hourglass constriction is thought to result from a swollen inflamed nerve becoming twisted with limb movement and being unable to return to a normal position. Hourglass constriction requires surgical intervention. If one sees neural swelling on transverse ultrasound scanning, longitudinal scanning should be performed to evaluate the presence of incomplete or complete hourglass constriction. Similarly, on MRI if the nerve changes in diameter abruptly along its course along with MRN signal
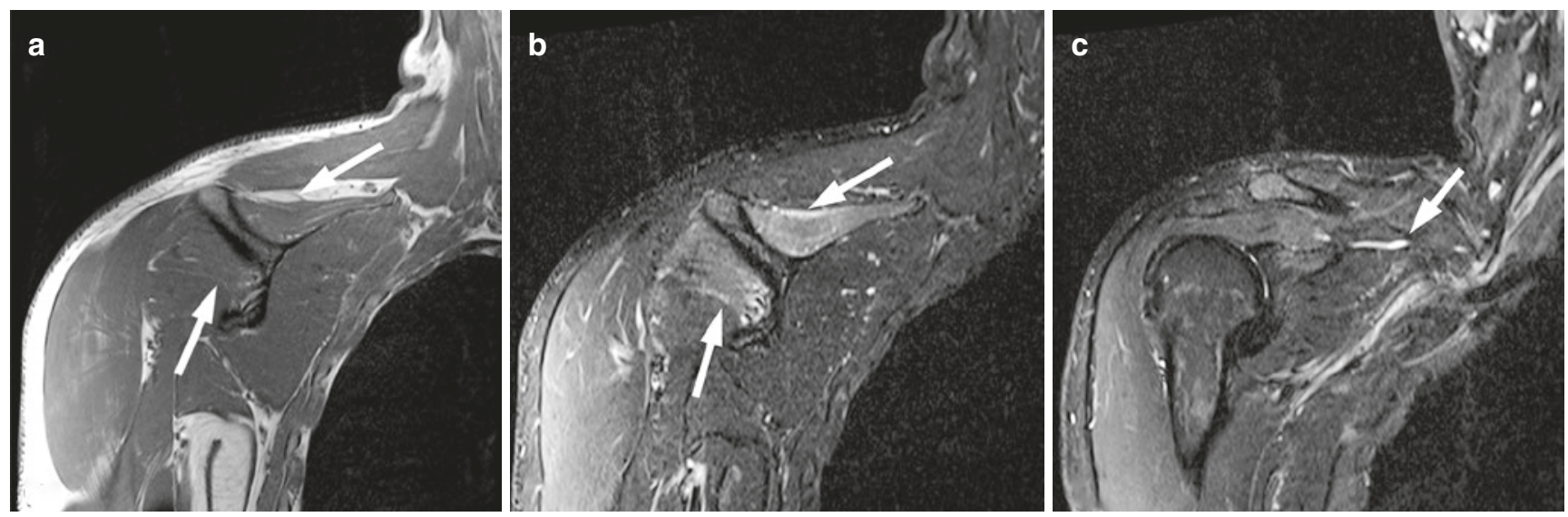

Fig. 18.6 In this patient with neuralgic amyotrophy, there is denervation oedema and moderate atrophy of the supraspinatus and infraspinatus muscles seen on both (a) T1-weighted (arrows) and (b) T2-weighted
STIR images (arrows). (c) The suprascapular nerve is slightly thickened with a slightly irregular calibre (arrow) highly suggestive of neuralgic amyotrophy 

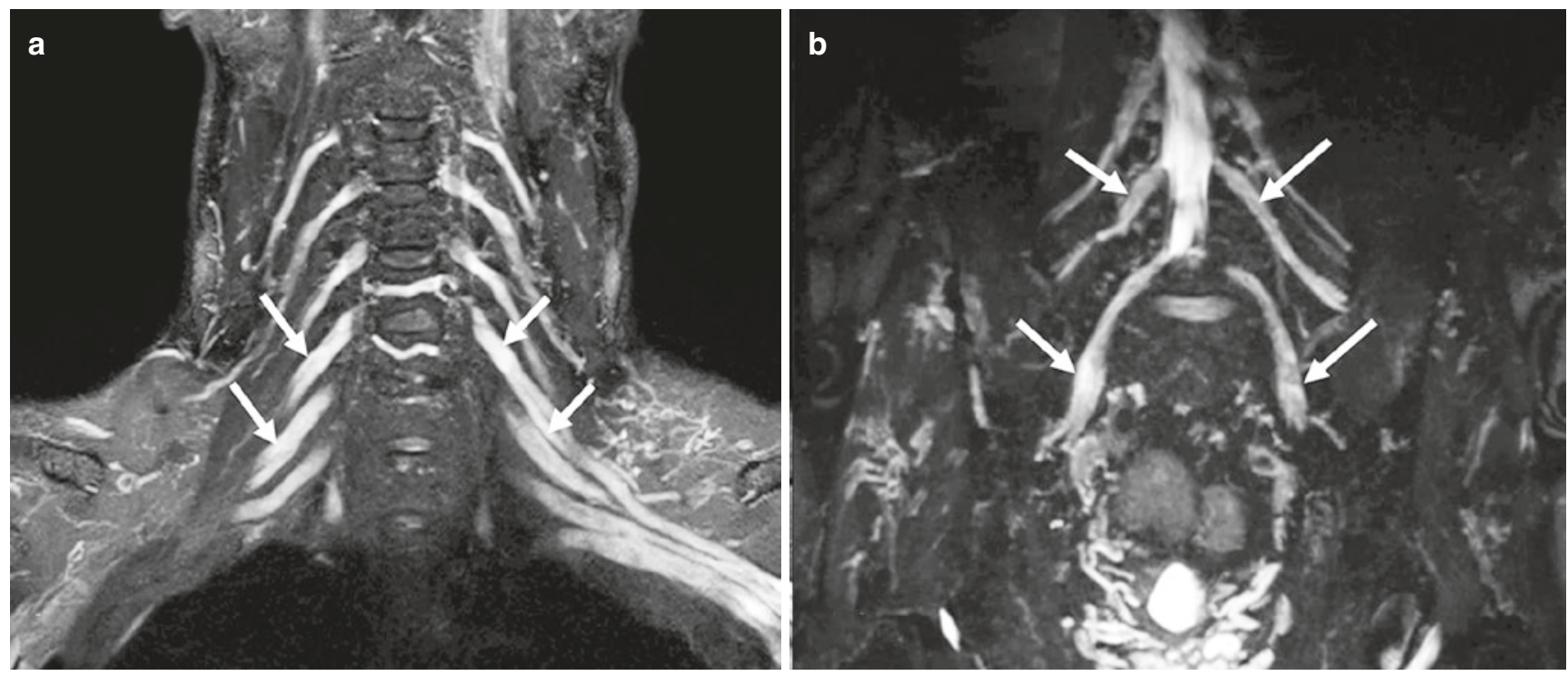

Fig. 18.7 MRN of patient with CIDP and characteristic enlargement of nerve roots and more peripheral plexal components on coronal MIP 3D SPACE STIR images, both in the (a) brachial (arrows) and (b) lumbosacral plexuses (arrows)

changes from hyper-, to hypo-, to hyperintensity, this may well represent focal hourglass constriction [22].

\section{Key Points}

- Neuralgic amyotrophy commonly affects the suprascapular nerve though extraplexal nerves are sometimes also affected.

- The imaging signs are neural swelling and, less frequently, fascicular entwinement \pm hourglass constriction of an affected swollen nerve.

- MR is more accurate than US at showing target muscle denervation.

\subsubsection{Inflammatory Neuropathy}

Inflammatory neuropathies are amenable to anti-inflammatory treatment. The two main types of inflammatory neuropathy are chronic inflammatory demyelinating polyneuropathy (CIDP) and multifocal motor neuropathy (MMN). CIDP is a mixed neuropathy while MMN is predominantly motor. Both upper and lower limbs tend to be affected. Ultrasound and MR features are increased neural size and muscle denervation \pm atrophy (Fig. 18.7). Any brachial trunk size of
$>8 \mathrm{~mm}^{2}$ and swelling of the median nerve $>13 \mathrm{~mm}^{2}$ in the arm or $>10 \mathrm{~mm}^{2}$ in the forearm is $99 \%$ specific for inflammatory neuropathy [23]. Multiple sites of nerve swelling may be present.

\section{Key Points}

- Imaging is helpful in assessing inflammatory neuropathy.

- Bilateral, diffuse, and multifocal involvement is common.

\subsection{Concluding Remarks}

One can appreciate that imaging of the peripheral nerves comprises a wide pathological spectrum. Imaging has greatly increased our knowledge and perception of peripheral nerve disorders enabling these disorders to be diagnosed and treated with greater clarity and precision. When imaging the peripheral nerves, one needs to be particularly mindful of the patient's history regarding previous trauma, malignancy, surgery, irradiation, as well as neurological symptoms and deficit. Ultrasound and MRI are, in many instances, complementary in this regard with considerable clinical practical benefit. 


\section{Take Home Messages}

- Ultrasound and MRI are incredibly helpful in the assessment of peripheral nerve disorders.

- High-resolution, targeted imaging is required.

- Usually either imaging modality will suffice, but to fully address some problems, both may be required and are usually complementary in this regard.

\section{References}

1. Filler AG, Maravilla KR, Tsuruda JS. MR neurography and muscle MR imaging for image diagnosis of disorders affecting the peripheral nerves and musculature. Neurol Clin. 2004;22:643-82.

2. Wasa J, Nishida Y, Tsukushi S, Shido Y, Sugiura H, Nakashima H, et al. MRI features in the differentiation of malignant peripheral nerve sheath tumors and neurofibromas. AJR Am J Roentgenol. 2010;194:1568-74.

3. Baumer P, Kele H, Kretschmer T, Koenig R, Pedro M, Bendszus $\mathrm{M}$, et al. Thoracic outlet syndrome in 3T MR neurography fibrous bands causing discernible lesions of the lower brachial plexus. Eur Radiol. 2014;24:756-61.

4. Menorca RM, Fussell TS, Elfar JC. Nerve physiology: mechanisms of injury and recovery. Hand Clin. 2013;29:317-30.

5. Stanisz GJ, Midha R, Munro CA, Henkelman RM. MR properties of rat sciatic nerve following trauma. Magn Reson Med. 2001;45:415-20.

6. Husarik DB, Saupe N, Pfirrmann CW, Jost B, Hodler J, Zanetti M. Elbow nerves: MR findings in 60 asymptomatic subjects - normal anatomy, variants, and pitfalls. Radiology. 2009;252:148-56.

7. Kästel T, et al. Magic angle effect: a relevant artifact in MR neurography a 3T? AJNR Am J Neuroradiol. 2016;32(5):821-7.

8. Chhabra A, Lee PP, Bizzell C, Soldatos T. 3 Tesla MR neurography - technique, interpretation, and pitfalls. Skelet Radiol. 2011;40:1249-60.

9. Vargas MI, Viallon M, Nguyen D, Beaulieu JY, Delavelle J, Becker M. New approaches in imaging of the brachial plexus. Eur J Radiol. 2010;74:403-10.

10. Madhuranthakam AJ, Lenkinski RE. Technical advancements in MR neurography. Semin Musculoskelet Radiol. 2015;19:86-93.

11. Kasper JM, Wadhwa V, Scott KM, Rozen S, Xi Y, Chhabra A. SHINKEI - a novel 3D isotropic MR neurography technique: technical advantages over 3DIRTSE-based imaging. Eur Radiol. 2015;25:1672-7.

12. Chhabra A, Soldatos T, Subhawong TK, Machado AJ, Thawait SK, Wang KC, et al. The application of three-dimensional diffusionweighted PSIF technique in peripheral nerve imaging of the distal extremities. J Magn Reson Imaging. 2011;34:962-7.

13. Guggenberger R, et al. Assessment of median nerve with MR neurography by using diffusion-tensor imaging: normative and pathologic diffusion values. Radiology. 2012;265:194-203.

14. Ng AWH, Griffith JF, Lee RKL, Tse WL, Wong CWY, Ho PC. Ultrasound carpal tunnel syndrome: additional criteria for diagnosis. Clin Radiol. 2018;73(2):214.e11-8.

15. Lee RKL, Griffith JF, Ng AWH, Tipoe GL, Chan AWH, Wong CWY, Tse WL, Ho PC. Cross-sectional area of the median nerve at the wrist: comparison of sonographic, MRI, and cadaveric measurements. J Clin Ultrasound. 2019;47(3):122-7.

16. Ng AWH, Griffith JF, Tong CSL, Law EKC, Tse WL, Wong CWY, Ho PC. MRI criteria for diagnosis and predicting severity of carpal tunnel syndrome. Skelet Radiol. 2020;49(3):397-405.

17. Ng AWH, Griffith JF, Tsai CSC, Tse WL, Mak M, Ho PC. MRI of the Carpal Tunnel 3 and 12 Months After Endoscopic Carpal Tunnel Release. AJR Am J Roentgenol. 2021;216(2):464-70.

18. Rhodes NG, Howe BM, Frick MA, Moran SL. MR imaging of the postsurgical cubital tunnel: an imaging review of the cubital tunnel, cubital tunnel syndrome, and associated surgical techniques. Skelet Radiol. 2019;48(10):1541-54.

19. Bäumer P, Dombert T, Staub F, Kaestel T, Bartsch AJ, Heiland S, Bendszus M, Pham M. Ulnar neuropathy at the elbow: MR neurography-nerve T2 signal increase and caliber. Radiology. 2011;260(1):199-206.

20. Ahlawat S, Blakeley JO, Langmead S, Belzberg AJ, Fayad LM. Current status and recommendations for imaging in neurofibromatosis type 1, neurofibromatosis type 2, and schwannomatosis. Skelet Radiol. 2020;49(2):199-219.

21. Chhabra A, Ahlawat S, Belzberg A, Andreseik G. Peripheral nerve injury grading simplified on MR neurography: as referenced to Seddon and Sunderland classifications. Indian J Radiol Imaging. 2014;24:217-24.

22. Sneag DB, Saltzman EB, Meister DW, Feinberg JH, Lee SK, Wolfe SW. MRI Bullseye sign: an indicator of peripheral nerve constriction in parsonage-turner syndrome. Muscle Nerve. 2017;56(1): 99-106.

23. Goedee HS, van der Pol WL, van Asseldonk JH, Franssen H, Notermans NC, Vrancken AJ, van Es MA, Nikolakopoulos S, Visser LH, van den Berg LH. Diagnostic value of sonography in treatment-naive chronic inflammatory neuropathies. Neurology. 2017;88(2):143-51.

Open Access This chapter is licensed under the terms of the Creative Commons Attribution 4.0 International License (http://creativecommons. org/licenses/by/4.0/), which permits use, sharing, adaptation, distribution and reproduction in any medium or format, as long as you give appropriate credit to the original author(s) and the source, provide a link to the Creative Commons license and indicate if changes were made.

The images or other third party material in this chapter are included in the chapter's Creative Commons license, unless indicated otherwise in a credit line to the material. If material is not included in the chapter's Creative Commons license and your intended use is not permitted by statutory regulation or exceeds the permitted use, you will need to obtain permission directly from the copyright holder. 